\title{
WALSH-FOURIER SERIES WITH COEFFICIENTS OF GENERALIZED BOUNDED VARIATION
}

\author{
F. MÓRICZ \\ (Received 23 February 1988) \\ Communicated by W. Moran
}

\begin{abstract}
We extend in different ways the class of null sequences of real numbers that are of bounded variation and study the Walsh-Fourier series of integrable functions on the interval $[0,1)$ with such coefficients. We prove almost everywhere convergence as well as convergence in the pseudometric of $L^{r}(0,1)$ for $0<r<1$.

1980 Mathematics subject classification (Amer. Math. Soc.) (1985 Revision): 42 C 10.

Keywords and phrases: Walsh-Paley system, coefficient sequence of bounded variation, pointwise convergence, convergence in $L^{r}(0,1)$-metric, $0<r<1$, arithmetic mean, de la Vallée Poussin mean, Dirichlet kernel, Fejêr kernel.
\end{abstract}

\section{Introduction}

We consider the Walsh orthonormal system $\left\{w_{k}(x): k=0,1, \ldots\right\}$ defined on the interval $[0,1)$ in the Paley enumeration (see, for example, [1, page 60]). We will study the Walsh-Fourier series

$$
\sum_{k=0}^{\infty} a_{k} w_{k}(x), \quad a_{k}=\int_{0}^{1} f(x) w_{k}(x) d x,
$$

of an integrable function $f \in L^{1}(0,1)$. In this paper, the integrals and the term "almost everywhere" (in abbreviation a.e.) are meant in the Lebesgue sense.

(C) 1989 Australian Mathematical Society $0263-6115 / 89 \$ A 2.00+0.00$

This research was completed while the author was a visiting professor at the University of Tennessee, Knoxville, during the academic year 1987/1988. 


\section{Main results}

We denote by

$$
s_{n}(f, x)=\sum_{k=0}^{n} a_{k} w_{k}(x) \quad(n=0,1, \ldots)
$$

the partial sums of series (1.1). Furthermore, we write

$$
\Delta a_{k}=a_{k}-a_{k+1}, \quad \Delta^{2} a_{k}=\Delta a_{k}-\Delta a_{k+1} \quad(k=0,1, \ldots)
$$

for the first and second differences, and set

$$
\lambda_{n}=[\lambda n] \quad(n=0,1, \ldots)
$$

where $\lambda$ is a fixed real number, $\lambda>1$, and $[\cdot]$ means the integral part.

THEOREM 1. If $f \in L^{1}(0,1)$ and

$$
\lim _{\lambda \rightarrow 1+0} \limsup _{n \rightarrow \infty} \frac{1}{\lambda_{n}-n+1} \sum_{k=n}^{\lambda_{n}}\left(\lambda_{n}-k+1\right)\left|\Delta^{m} a_{k}\right|=0
$$

for $m=1$ or 2 , then

$$
\lim _{n \rightarrow \infty} s_{n}(f, x)=f(x) \text { a.e. }
$$

THEOREM 2. If $f \in L^{1}(0,1)$ and condition (2.2) is satisfied for $m=1$ or 2 , then

$$
\lim _{n \rightarrow \infty} \int_{0}^{1}\left|s_{n}(f, x)-f(x)\right|^{r} d x=0 \text { for } 0<r<1 / m .
$$

Clearly, if condition (2.2) is satisfied for $m=1$, then it is automatically satisfied for $m=2$, but the converse implication fails in general.

We draw two corollaries of Theorems 1 and 2.

Corollary 1. If $f \in L^{1}(0,1)$ and

$$
\lim _{\lambda \rightarrow 1+0} \limsup _{n \rightarrow \infty} \sum_{k=n}^{\lambda_{n}}\left|\Delta^{m} a_{k}\right|=0
$$

for $m=1$ or 2, then we have conclusions (2.3) and (2.4).

EXAMPLE. Let $\lambda=\lambda^{(j)}=1+2^{-j}$ for $j=1,2, \ldots$ and consider the sequence $\left\{a_{k}\right\}$ defined as follows:

$$
\Delta^{m} a_{k}= \begin{cases}\frac{(-1)^{j}}{j\left(2^{2 j}+2^{j}-k+1\right)} & \text { if } 2^{2 j} \leq k \leq 2^{2 j}+2^{j} \text { for some } j=1,2, \ldots \\ 0 & \text { otherwise. }\end{cases}
$$


It is not hard to check that $\left\{a_{k}\right\}$ is a null sequence and condition (2.2) is satisfied, but condition (2.5) is not. This example shows that Theorem 1 is more general than Corollary 1.

Corollary 1 applies to many particular cases. We refer the reader to [2] where seven main cases and even further subcases are listed and discussed in details. We present here one more special case of (2.5) which is not contained in [2].

COROLlary 2. If $f \in L^{1}(0,1)$ and the finite limit

$$
\lim _{n \rightarrow \infty} \frac{1}{n} \sum_{k=1}^{n} k\left|\Delta^{m} a_{k}\right|=L
$$

exists for $m=1$ or 2 , then we have conclusions (2.3) and (2.4).

We recall that a sequence $\left\{a_{k}\right\}$ is said to be of bounded variation if

$$
\sum_{k=0}^{\infty}\left|\Delta a_{k}\right|<\infty
$$

Obviously, if $\left\{a_{k}\right\}$ is of bounded variation, then (2.6) is satisfied with $m=1$ (and a fortiori with $m=2$ ) and $L=0$. Thus, each of the conditions (2.2), (2.5) and (2.6) for either $m=1$ or $m=2$ can be considered a generalization of the notion of bounded variation.

We note that the counterpart of Corollary 1 for trigonometric Fourier series was proved by Chen [2], while that of Corollary 2 was proved by Stanojevic [5].

\section{Proofs}

We denote by

$$
\sigma_{n}(f, x)=\frac{1}{n+1} \sum_{j=0}^{n} s_{j}(f, x) \quad(n=0,1, \ldots)
$$

the first arithmetic means of series (1.1). It is well-known (see [3] and [4], respectively) that if $f \in L^{1}(0,1)$, then

$$
\lim _{n \rightarrow \infty} \sigma_{n}(f, x)=f(x) \text { a.e. }
$$

and

$$
\lim _{n \rightarrow \infty} \int_{0}^{1}\left|\sigma_{n}(f, x)-f(x)\right| d x=0
$$


Next, we consider the so-called generalized de la Vallée Poussin means defined by

$$
\tau_{n}(f, \lambda, x)=\frac{1}{\lambda_{n}-n+1} \sum_{j=n}^{\lambda_{n}} s_{j}(f, x)
$$

where $\lambda>1$ and $\lambda_{n}$ is given by $(2.1)$.

Using the representation

$$
\tau_{n}(f, \lambda, x)=\frac{\lambda_{n}+1}{\lambda_{n}-n+1} \sigma_{\lambda_{n}}(f, x)-\frac{n}{\lambda_{n}-n+1} \sigma_{n-1}(f, x),
$$

we have from (3.1) and (3.2) that for any fixed $\lambda>1$,

$$
\lim _{n \rightarrow \infty} \tau_{n}(f, \lambda, x)=f(x) \text { a.e. }
$$

and

$$
\lim _{n \rightarrow \infty} \int_{0}^{1}\left|\tau_{n}(f, \lambda, x)-f(x)\right| d x=0 .
$$

Remark 1. Actually, we have (3.3) at each point $x$, at which (3.1) is satisifed, Furthermore, if the convergence of $\sigma_{n}(f, x)$ is uniform on a certain set $E$, then the convergence of $\tau_{n}(f, \lambda, x)$ is also uniform on $E$ for fixed $\lambda$.

Proof of Theorems 1 AND 2 FOR $m=1$. By definition,

$$
\tau_{n}(f, \lambda, x)-s_{n}(f, x)=\frac{1}{\lambda_{n}-n+1} \sum_{j=n+1}^{\lambda_{n}} \sum_{k=n+1}^{j} a_{k} w_{k}(x) .
$$

For each $j \geq n+2$, a summation by parts yields

$$
\sum_{k=n+1}^{j} a_{k} w_{k}(x)=-a_{n+1} D_{n}(x)+\sum_{k=n+1}^{j-1} D_{k}(x) \Delta a_{k}+a_{j} D_{j}(x)
$$

where

$$
D_{n}(x)=\sum_{k=0}^{n} w_{k}(x) \quad(n=0,1, \ldots)
$$

is the Dirichlet kernel for the Walsh system. It is well known (see, for example, [3]) that

$$
\left|D_{n}(x)\right|<2 / x \quad(n=0,1, \ldots ; 0<x<1) .
$$

From this, (3.5) and (3.6), a simple computation gives that for $0<x<1$,

$$
\begin{aligned}
& \left|\tau_{n}(f, \lambda, x)-s_{n}(f, x)\right| \\
& \quad \leq \frac{2}{\left(\lambda_{n}-n+1\right) x} \sum_{j=n+1}^{\lambda_{n}}\left(\left|a_{n+1}\right|+\sum_{k=n+1}^{j-1}\left|\Delta a_{k}\right|+\left|a_{j}\right|\right) \\
& \quad=\frac{o(1)}{x}+\frac{2}{\left(\lambda_{n}-n+1\right) x} \sum_{k=n+1}^{\lambda_{n}-1}\left(\lambda_{n}-k\right)\left|\Delta a_{k}\right|,
\end{aligned}
$$


where $o(1)$ does not depend on $x$. Here we used the fact that $f \in L^{1}(0,1)$ implies that

$$
\lim _{n \rightarrow \infty} a_{n}=0
$$

By (2.2) and (3.8), for every $0<x<1$,

$$
\lim _{\lambda \rightarrow 1+0} \limsup _{n \rightarrow \infty}\left|\tau_{n}(f, \lambda, x)-s_{n}(f, x)\right|=0
$$

and for every $0<r<1$,

$$
\lim _{\lambda \rightarrow 1+0} \limsup _{n \rightarrow \infty} \int_{0}^{1}\left|\tau_{n}(f, \lambda, x)-s_{n}(f, x)\right|^{r} d x=0 .
$$

Combining (3.3) and (3.10) yields (2.3), while combining (3.4) and (3.11) yields (2.4) in the case $m=1$.

REMARK 2. It is easy to see that the convergence in (3.10) is uniform on any interval $[\delta, 1)$ with $0<\delta<1$.

Proof of Theorems 1 AND 2 fOR $m=2$. We perform one more summation by parts on the right-hand side of (3.6), which results in the following:

$$
\begin{aligned}
\sum_{k=n+1}^{j} a_{k} w_{k}(x) & =-a_{n+1} D_{n}(x)-(n+1) F_{n}(x) \Delta a_{n+1} \\
& +\sum_{k=n+1}^{j-2}(k+1) F_{k}(x) \Delta^{2} a_{k}+j F_{j-1}(x) \Delta a_{j-1}+a_{j} D_{j}(x)
\end{aligned}
$$

where

$$
F_{n}(x)=\frac{1}{n+1} \sum_{j=0}^{n} D_{j}(x) \quad(n=0,1, \ldots)
$$

is the Fejer kernel for the Walsh system.

According to Fine [3], for all positive integers $n$ and $m$, and for all $x$, except possibly for a dyadic rational $x$,

$$
(n+1)\left|F_{n}(x)\right|<\frac{4}{x\left(x-2^{-m}\right)}+\frac{4}{x^{2}}=C(x) \text { if } 2^{-m}<x<2^{-m+1} .
$$


It follows from (3.5), (3.7), (3.9), (3.12) and (3.13) that for all $0<x<1$, except perhaps the dyadic rationals,

$$
\begin{aligned}
& \left|\tau_{n}(f, \lambda, x)-s_{n}(f, x)\right| \\
& \leq \frac{1}{\lambda_{n}-n+1} \sum_{j=n+1}^{\lambda_{n}}\left\{\frac{2}{x}\left(\left|a_{n+1}\right|+\left|a_{j}\right|\right)\right. \\
& \left.+C(x)\left(\left|\Delta a_{n+1}\right|+\sum_{k=n+1}^{j-2}\left|\Delta^{2} a_{k}\right|+\left|\Delta a_{j-1}\right|\right)\right\} \\
& =\left(\frac{1}{x}+C(x)\right) o(1)+\frac{C(x)}{\lambda_{n}-n+1} \sum_{k=n+1}^{\lambda_{n}-2}\left(\lambda_{n}-k-1\right)\left|\Delta^{2} a_{k}\right|,
\end{aligned}
$$

where $o(1)$ does not depend on $x$.

This and (2.2) imply that for all $x$, except the dyadic rationals, we have (3.10).

Let $0<r<1 / 2$. By $(3.14)$,

$$
\begin{aligned}
& \int_{0}^{1}\left|\tau_{n}(f, \lambda, x)-s_{n}(f, x)\right|^{r} d x \\
& \quad=o(1)+\left\{o(1)+\frac{1}{\lambda_{n}-n+1} \sum_{k=n+1}^{\lambda_{n}-2}\left(\lambda_{n}-k-1\right)\left|\Delta^{2} a_{k}\right|\right\} \int_{0}^{1} C^{r}(x) d x .
\end{aligned}
$$

By (3.13),

$$
\begin{aligned}
\int_{0}^{1} C^{r}(x) d x & \leq \sum_{m=1}^{\infty} \int_{2^{-m}}^{2^{-m+1}} \frac{4^{r}}{x^{r}\left(x-2^{-m}\right)^{r}} d x+\int_{0}^{1} \frac{4^{r}}{x^{2 r}} d x \\
& \leq \sum_{m=1}^{\infty} \frac{4^{r}}{1-r} 2^{m(2 r-1)}+\frac{4^{r}}{1-2 r}<\infty
\end{aligned}
$$

Putting the last two estimates together gives (3.11) for $0<r<1 / 2$.

Finally, combining (3.3) and (3.10) yields (2.3), while combining (3.4) and (3.11) yields (2.4) in the case $m=2$.

Proof of Corollary 2. It is enough to show that condition (2.6) implies (2.5). Clearly,

$$
\begin{aligned}
\sum_{k=n}^{\lambda_{n}}\left|\Delta^{m} a_{k}\right| & \leq \frac{1}{n} \sum_{k=n}^{\lambda_{n}} k\left|\Delta^{m} a_{k}\right| \\
& \leq \frac{\lambda_{n}}{n} \frac{1}{\lambda_{n}} \sum_{k=1}^{\lambda_{n}} k\left|\Delta^{m} a_{k}\right|-\frac{n-1}{n} \frac{1}{n-1} \sum_{k=1}^{n-1} k\left|\Delta^{m} a_{k}\right| .
\end{aligned}
$$


Given any $\varepsilon>0$, by (2.6) we have

$$
\begin{aligned}
\sum_{k=n}^{\lambda_{n}}\left|\Delta^{m} a_{k}\right| & \leq \frac{\lambda_{n}}{n}(L+\varepsilon)-\frac{n-1}{n}(L-\varepsilon) \\
& =\frac{\lambda_{n}-n+1}{n} L+\frac{\lambda_{n}+n-1}{n} \varepsilon
\end{aligned}
$$

provided $n$ is large enough. Thus, it follows from (2.1) and (3.15) that

$$
\limsup _{n \rightarrow \infty} \sum_{k=n}^{\lambda_{n}}\left|\Delta^{m} a_{k}\right| \leq(\lambda-1) L+(\lambda+1) \varepsilon .
$$

Since $\varepsilon>0$ is arbitrary, hence we get $(2.5)$.

\section{Concluding remarks}

It turns out from the proofs of Theorems 1 and 2 that we can also deduce (2.3) and (2.4) when the "lim sup" in (2.2) equals zero for a specific value of $\lambda>1$. Here we formulate only the case $\lambda=2$.

THEOREM 3. If $f \in L^{1}(0,1)$ and

$$
\lim _{n \rightarrow \infty} \frac{1}{n+1} \sum_{k=n}^{2 n}(2 n-k+1)\left|\Delta^{m} a_{k}\right|=0
$$

for $m=1$ or 2, then we have conclusions (2.3) and (2.4).

Condition (4.1) is also a generalization of the notion of bounded variation. Another by-product of the proof of Theorem 1 relates to continuous functions.

THEOREM 4. If $f \in C[0,1)$ and condition (2.2) is satisfied for $m=1$, then for every $0<x<1$,

$$
\lim _{n \rightarrow \infty} s_{n}(f, x)=f(x),
$$

and this convergence is uniform on each interval $[\delta, 1)$ with $0<\delta<1$.

Relation (4.2) is an immediate consequence of Remarks 1 and 2 in Section 3 and the following well-known result (see [3]): if $f \in C[0,1$ ), then for every $x$

$$
\lim _{n \rightarrow \infty} \sigma_{n}(f, x)=f(x)
$$

and this convergence is uniform on the whole interval $[0,1)$. 
We note that the counterpart of Theorem 4 for trigonometric Fourier series was proved by Chen [2].

\section{References}

[1] G. Alexits, Convergence problems of orthogonal series (Pergamon Press, Oxford, 1961).

[2] Chang-Pao Chen, 'Pointwise convergence of trigonometric series,' J. Austral. Math. Soc. Ser. A 43 (1987), 291-300.

[3] N. J. Fine, 'On the Walsh functions,' Trans. Amer. Math .Soc. 65 (1949), 372-414.

[4] G. Morgenthaler, 'Walsh-Fourier series,' Trans. Amer. Math. Soc. 84 (1957), 472-507.

[5] V. B. Stanojevic, 'Convergence of Fourier series with complex quasimonotone coefficients and coefficients of bounded variation of order m, J. Math. Anal. Appl. 115 (1986), 482505.

\section{Bolyai Institute}

University of Szeged

Aradi Vertanuk Tere 1

6720 Szeged

Hungary 